\title{
Game Theory Modelling for Vehicle U-Turn Behavior and Simulation Based on Cellular Automata
}

\author{
WenJing Wu $\mathbb{D}^{\mathbb{1}},{ }^{1}$ ZhiKang Liang $\mathbb{D},{ }^{1}$ QingYu Luo $\mathbb{D}^{1},{ }^{1}$ and FangWu $\mathrm{Ma}^{2}$ \\ ${ }^{1}$ School of Transportation, Jilin University, Changchun, China \\ ${ }^{2}$ College of Automotive Engineering, Jilin University, Changchun, China
}

Correspondence should be addressed to QingYu Luo; luoqy@jlu.edu.cn

Received 13 April 2018; Accepted 31 July 2018; Published 23 August 2018

Academic Editor: Paolo Renna

Copyright (C) 2018 WenJing Wu et al. This is an open access article distributed under the Creative Commons Attribution License, which permits unrestricted use, distribution, and reproduction in any medium, provided the original work is properly cited.

In order to analyze the effect of U-turn vehicle on traffic performance, the work develops a game theory-based description of drivers' interactions in U-turn scene, considering the decision-making uncertainty. The hybrid strategy of the game is obtained. The relevant parameters of model are calibrated by collected video data in Changchun, China. A two-way four-lane cellular automaton model with the game model imbedded is constructed for identifying the effect of U-turn vehicle on traffic performance. The influencing factors are identified with their correlation analyzed by numerical simulation of different traffic conditions. According to the simulation results, U-turn traffic has a significant influence on traffic delay in the lane of same direction, compared with opposite direction. The severity of conflict between vehicles is classified and the causes are identified by analyzing the arrival rate of the U-turn vehicle and the conflicting straight vehicle and the relationship with one another. In addition, the threshold of traffic flow causing traffic conflict and traffic delay are proposed. The results show that the proposed models reconstructed the traits of traffic flow and conflict phenomenon in the presence of $\mathrm{U}$-turn vehicles on road section.

\section{Introduction}

The road safety has become a high-priority issue to traffic engineers and traffic authorities for decades. Conflicts between different participants, including cars, motors, buses, bicycles, and pedestrians [1-4] in the traffic, reduce road safety, resulting in extra travelling time. To solve the related problems, a number of studies have been carried out either by crash based methods such as the historical crash data analysis [5-7], or by proactive (noncrash) based methods which include conflicts as well as traffic maneuvers studies [8-12]. Also some research on in-vehicle alerts techniques such as the action detection or recognition technology is included [13-15]. Many highlighted works have adequately addressed modelling and simulation of traffic conflict phenomenon such as vehicle-pedestrian in crosswalk and vehicle-vehicle in intersection. The focus of this paper is vehicle-vehicle in U-turn, an essential conflict phenomenon in heavy traffic.

U-turn facilities are used as open areas for two-way traffic flow on the road, often set at the entrance of the intersection or the middle of the road section. U-turn vehicles have significant impact on the traffic performance. Median openings (including U-turns) are considered as the crashprone locations of Thai highways [16]. The type of U-turn facilities and its impacts on the traffic are studied. Meel [17] studied the safety of four types of layout design of U-turn on the divided Thai highways. Della [18] studied the effect of U-turn facilities on traffic delays and travel time. Olarte [19] analyzed the safety for rural inconspicuous restricted crossing U-turn intersections by density models. Liu [20] studied the safety effects of the separation distances between driveway exits and downstream U-turn locations, using the reported crash dates. Many studies have been carried out to investigate the $\mathrm{U}$-turn vehicle interference and effect on macroscopic traffic performance. However, driver-driver interactions characteristics perceived from a microscopic viewpoint have not been given enough attention. It is helpful to understand the mechanism of vehicle conflict or traffic jam, thus preventing accidents.

In theory, straight vehicle should get priority over U-turn vehicle all the time. The gap acceptance mechanism of U-turn vehicle is the process of selecting suitable vehicle gap after 


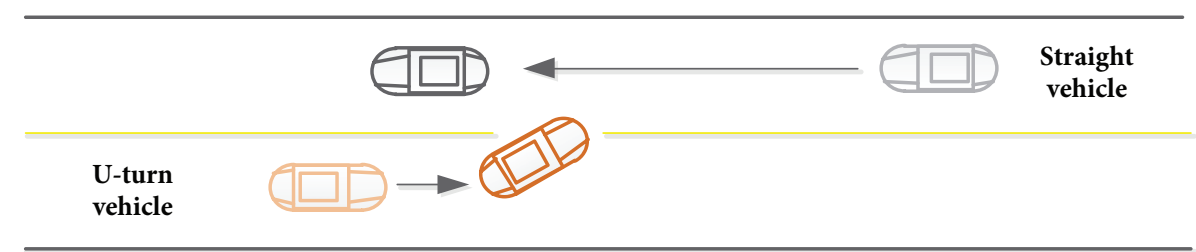

(a)

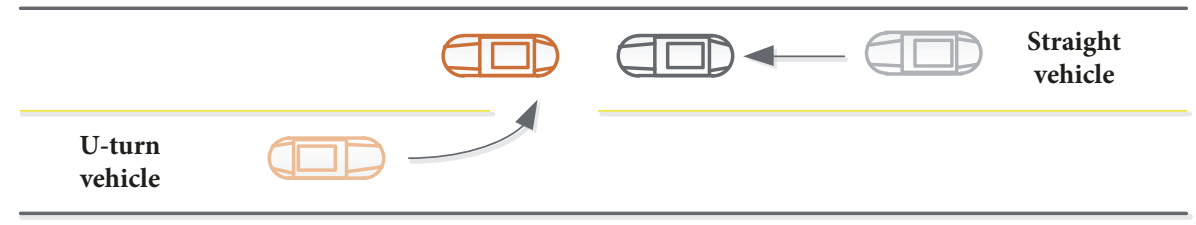

(b)

FIgURE 1: U-turn scenario. (a) The U-turn vehicle yields. (b) The straight vehicle yields.

arriving at a mid-block median opening. In this process, the U-turn vehicle may be successful or unsuccessful, depending on the available gaps appearance of straight vehicle. In reality, the U-turning vehicles often do not wait for an acceptable gap in the on-coming straight-traffic. They gradually move onto the conflicting lane to show their intention to go; particularly when the U-turn traffic is in a long queue or has waited for a long time, the drivers tend to be more aggressive. At the same time, the conflicting straight vehicle will be reluctant to yield by increasing speed, changing lanes, or flashing their headlights. The interaction between Uturn vehicle and straight vehicle can be described as two persons' noncooperative game behavior. Game theory has the advantage of take into account the impact of stochasticity and decision uncertainty, providing a powerful tool for analyzing the interaction behavior in transport system. Game theory has been applied widely in different disciplines, such as lane changing, car-following, and driverless vehicle control [21-25]. The work considered the traffic delay and threat perception as the factors of the game, constructing a $\mathrm{U}$ turn game (UTG) model to solve the problem of decisionmaking uncertainty between U-turning vehicle and straight vehicle. We explain the effectiveness of the game model straight empirical data in Changchun, China, and calibrate the relevant parameters of the game model.

In addition, there have been extensive studies done on the simulation of the vehicle-vehicle interaction. The most popular simulation model is rule-based models [26, 27], which defined some fixed parameters beforehand, such as acceptance gaps, time to collision (TTC), and grading of severity of conflicts [28-30]. Actually, those rule-based models are not suitable for reflecting the random nature of traffic conflict, because the decision of the drivers is subject to the objective effect of the intervening gap and the subjective effect of the driver's risk and delay perception. Cellular Automata model can overcome those defects. Cellular Automata model is a mathematical one with discrete time and space units, widely used in the interpretation of the complex nonlinear phenomena of traffic system through the modification of rules [31-33]. So, it is suitable to simulate U-turn behavior and reflect its impact on the traffic performance. In the work, we establish a two-way four-lane cellular automata model with U-turn facility in the middle. The hybrid strategy of the game is embedded into the cellular automata model, as the rule of U-turn.

This paper makes two contributions. The first is quantifying the dynamic process of decision-making varied with traffic character over each lane at U-turn road section. The second contribution is evaluating the impact of the relationship between the arriving rate of U-turn vehicles on one side and the arriving rate of straight vehicles on opposite side lane on the lane-based delay and vehicle-vehicle conflict as well as exploring the threshold of different levels of traffic delay and conflict severity, which would be helpful for transportation agencies to meet driver's time-cost and safety or comfort needs when they choose the opening for U-turn vehicles in road section.

The work is organized as follows. In Section 2, a game model is established, considering the uncertainty of drivers' decisions. Section 3 presents the cellular automata system model, with game model embedded, for modelling the effect of U-turn vehicle on traffic performance. After that, the effects of different traffic variables on the U-turn section traffic performance are studied in the extensive traffic simulations. Section 4 shows the results. Section 5 refers to the conclusions.

\section{UTG Model}

A typical U-turn scenario is shown in Figure 1. The U-turn vehicle wants to turn to the opposite lanes while the straight vehicle is coming from the opposite direction. They have the same opportunities to cross the U-turn place. Figure 1(a) shows that the straight vehicle goes first and the U-turn vehicle is waiting. Figure 1(b) shows that the U-turn vehicle goes first and the straight vehicle is waiting. The decisions of the drivers are subject to the opportunity of the intervening gap and also influenced by driver's risk and delay perception, which leads to the uncertainty of decision-making behavior; that is, the decision of the drivers is not a clear choice 
TABLE 1: Game payoff matrix.

\begin{tabular}{lccr}
\hline & & U-turn vehicle \\
& & Pass & Wait \\
\hline \multirow{2}{*}{ Straight vehicle } & Pass & $-g_{2}-a c h_{2},-g_{1}-a c h_{1}$ & $g_{2}+a h_{2}, g_{1}-a h_{1}$ \\
& Wait & $g_{2}-a h_{2}, g_{1}+a h_{1}$ & $g_{2}-a h_{2}, g_{1}-a h_{1}$ \\
\hline
\end{tabular}

TABLE 2: Game payoff matrix quantified.

\begin{tabular}{lccr}
\hline & & & U-turn vehicle \\
& & Pass & Wait \\
\hline & Pass & $-k-a c t_{1}$, & $k+a t_{1}$, \\
Straight vehicle & & $-k-a c t_{2}$, & $k-a t_{2}$ \\
& Wait & $k-a t_{1}$, & $k t_{1}$, \\
& & $k+a t_{2}$ & $k-a t_{2}$ \\
\hline
\end{tabular}

strategy. We can only get a probability of the driver's different decisions.

\subsection{Model Architecture}

2.1.1. Assumption. Firstly, we formulate the game, taking into account the players, strategy, and payoff.

In this game theory model, there are two players, $i=1,2$, wherein $i=1$ represents the U-turn vehicle's driver, and $i=$ 2 is straight vehicle's driver. As rational persons, both sides expect to maximize their own interests. Their strategies are both set \{pass, wait\}.

Table 1 shows the game theory model of U-turn. $h_{i}$ and $g_{i}$ are used for the utility of delay and the utility of threat perception; $a$ is the weight coefficient. When both the U-turn vehicle and the straight vehicle want to pass, an emergency brake would happen, both parties would lose the utility of delay and the utility of threat perception. We introduce $c$ as a multiplier for the utility of delay in that situation. When the U-turn vehicle wants to pass and the straight vehicle wants to wait, the U-turn vehicle will obtain the utility of delay and the utility of threat perception, and the straight vehicle gains threat perception utility but loses delay utility due to waiting, with the same utility setting on the contrary situation. When both actors want to wait, they both will gain threat perception utility but lose delay utility due to waiting.

\subsubsection{Analysis of Payment Function}

(1) Delay of the U-Turn Vehicle. When the straight vehicle is the first to approach, the U-turn vehicle will stop to wait, and the delay time of the U-turn vehicle is the time to wait for the straight vehicle to pass. $t_{1}$ denotes the delay of the U-turn vehicle when encountering the straight vehicle.

(2) Delay of the Straight Vehicle. When the U-turn vehicle is the first to pass, the straight vehicle will stop to wait. The delay of the straight vehicle $t_{2}$ is the time that the straight vehicle waits for the U-turn vehicle to pass the conflict point.
(3) Threat Perception. It is assumed that smaller intervening gap results in the greater driver's threat. The threat is inversely proportional to the distance between vehicles and proportional to the speed, so $k=v / L$ is used to express the threat perception. Table 2 shows the quantified game payoff matrix.

2.2. Solution of the UTG Model. As shown in Table 2, we can obtain this game with two dominant game strategies\{the straight vehicle pass, the U-turn vehicle waits\} and \{the straight vehicle waits, the U-turn vehicle pass\}. From a stability point of view, the two strategic combinations have advantage for each other, so they are unstable in the evolution process. Based on the Nash Equilibrium principle, there exists a mixed dominance strategy combination in the solution of the model. The solution is Nash Equilibrium, and the results are shown in (1) and (2). The mixed strategy Nash Equilibrium can be interpreted as a stochastic steady state.

U-turn vehicle (U-turn, wait) probability is as follows:

$$
\left(p_{u u}, p_{u w}\right)=\left(\frac{2 a t_{1}}{2 k+(1+c) a t_{1}}, 1-\frac{2 a t_{1}}{2 k+(1+c) a t_{1}}\right)
$$

Straight vehicle (travel, wait) probability is as follows:

$$
\left(p_{s t}, p_{s w}\right)=\left(\frac{2 a t_{2}}{2 k+(1+c) a t_{2}}, 1-\frac{2 a t_{2}}{2 k+(1+c) a t_{2}}\right)
$$

From the perspective of social optimal efficiency, U-turn vehicles and straight vehicles should be polite in the U-turn area; that is, the straight vehicle pass, the U-turn vehicle waits\} and \{the straight vehicle waits, the U-turn vehicle pass\} are what we hope to see. \{the straight vehicle pass, the Uturn vehicle pass\} is likely to cause traffic safety risks; $\{$ the straight vehicle waits, the U-turn vehicle waits\} reduces the efficiency of two players of the game. However, according to Nash Equilibrium, there is still a certain probability that both players of the game will choose the latter two strategies. At this time, the vehicle decelerates and brings turbulence in the original traffic flow, resulting in a decrease in traffic 


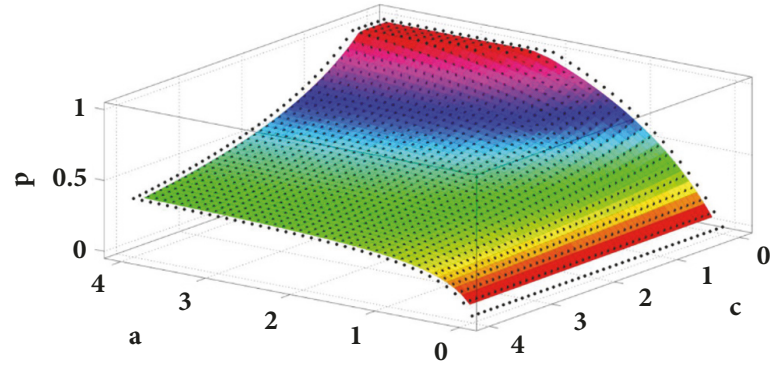

(a)

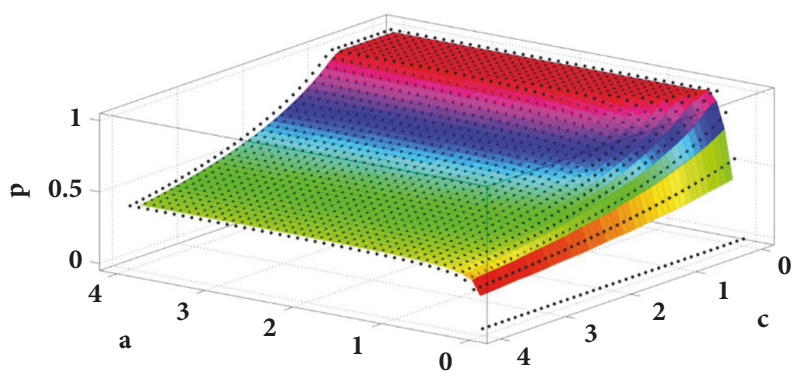

(b)

Figure 2: The relationship between the variables and the Nash Equilibrium solution. (a) The headway is equal to 1. (b) The headway is equal to 2.75 .

efficiency. The premise of the previous U-turn model to deal with whether the driver makes a U-turn is that the U-turn vehicle does not affect the following vehicle after U-turn, but in practice the drivers expect to go ahead because of egoism, which triggers a game strategy that is not conducive to the efficiency of traffic flow and makes the social dilemma structure in the U-turn behavior. The U-turn game is the same as the lane changing game [34-36]; there are social dilemmas hidden behind the traffic flow.

Tanimoto et al. [34-36] studied the types of social dilemma structures under different traffic flow densities. The difference is that we studied the types of social dilemma structures under the Nash Equilibrium influenced by various variables.

According to (1) and (2), the variables that affect the Nash Equilibrium solution include delay weight coefficient $a$, threat perception $k$, postconflict delay multiple $c$, and vehicle delay $t$. Since $k$ and $t$ are related to the vehicle headway, $k$ and $t$ are measured by the headway. Figure 2 shows the relationship between the variables and the Nash Equilibrium solution. The ordinate is the probability that the U-turn vehicle or the straight vehicle passes.

The social dilemma structure can be divided into Prisoner's dilemma and Chicken dilemma. Prisoner's dilemma indicates that Nash Equilibrium is close to one of the pure strategic situations. At this time, the probability that a certain strategy appears is close to 1 . Chicken dilemma has the characteristics of polymorphic Nash Equilibrium, not close to any pure strategic situation; it means that no strategy has a probability close to 1 . Through the analysis of Figure 2, it can be found that under the influence of different variables the probability of U-turn vehicles or straight vehicles pass is different, indicating that the type of social dilemma in the U-turn game is different. (1) When the value of headway is small, $a$ and $c$ affect the size of the Nash Equilibrium solution. When $a>2, c<0.5$, the social dilemma structure has the opportunity to become Prisoner's dilemma, and the other situation is Chicken dilemma. (2) When the value of headway is large, $c$ affects the size of the Nash Equilibrium solution. When $c<1$, the social dilemma structure has the opportunity to become Prisoner's dilemma, and the other situation is Chicken dilemma. Reflected in the real scene, the size of the headway represents the size of the traffic flow density;

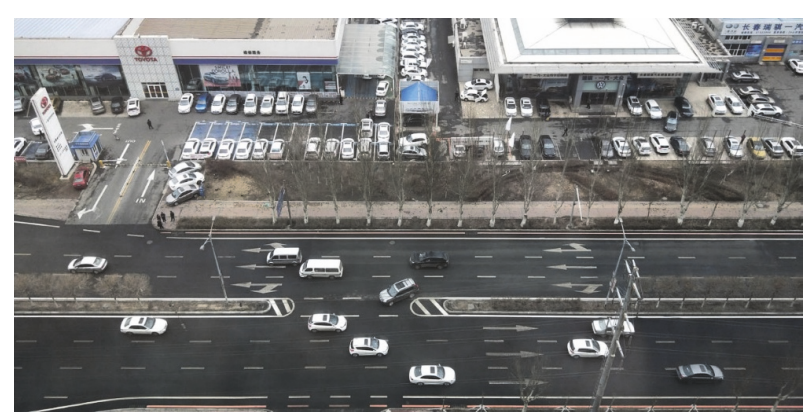

Figure 3: Scene of the U-turn point in Changchun, China.

the greater the traffic flow density, the smaller the headway. The delay weight coefficient $a$ represents the driver's attitude towards delay in the region. The more impulsive the driver is, the more he values the time and the greater $a$. The postconflict delay multiple $c$ represents the enforcement of the posttraffic conflicts in the region. The greater the enforcement, the larger $c$. Therefore, in the real scene, when the traffic flow density is constant, the driver is more impulsive, the law enforcement is smaller, and the social dilemma structure is more likely to change from Chicken dilemma to Prisoner's dilemma.

2.3. Empirical Verification. We conducted an empirical survey at the U-turn point (at the east side of the intersection of Guanghua Street and Weishan Road in Changchun City, China), as shown in Figure 3. We collected video data on November 2, 2017. Each game between U-turn vehicles and straight vehicles is recorded, like their gap acceptance behaviors and their speeds, headway interval, and so on.

2.3.1. Samples of Games. A total of 38 vehicles preparing for U-turn in road section are selected as samples in this research, and there are 198 games shown in Figure 4. The abscissa represents each game and the ordinate represents its corresponding headway of straight vehicle in opposite lane in each game. The different colors of the points represent different results. The blue points represent the $U$-turn vehicle turned and the red points represent the U-turn vehicle waiting. The results show that the behavior of vehicles is 
TABLE 3: Headway statistics in different scenes.

\begin{tabular}{lcccc}
\hline Headway interval & $\begin{array}{c}\text { The number of } \\
\text { turning vehicles }\end{array}$ & $\begin{array}{c}\text { The number of } \\
\text { waiting vehicles }\end{array}$ & Total by row & $\begin{array}{c}\text { U-turn vehicle } \\
\text { (U-turn, wait) } \\
\text { probability }\end{array}$ \\
\hline$(0-2]$ & 24 & 150 & 174 & $(0.14,0.86)$ \\
\hline$(2-3.5]$ & 14 & 10 & 24 & $(0.58,0.42)$ \\
\hline Total by column & 38 & 160 & 198 & $(0.19,0.81)$ \\
\hline
\end{tabular}

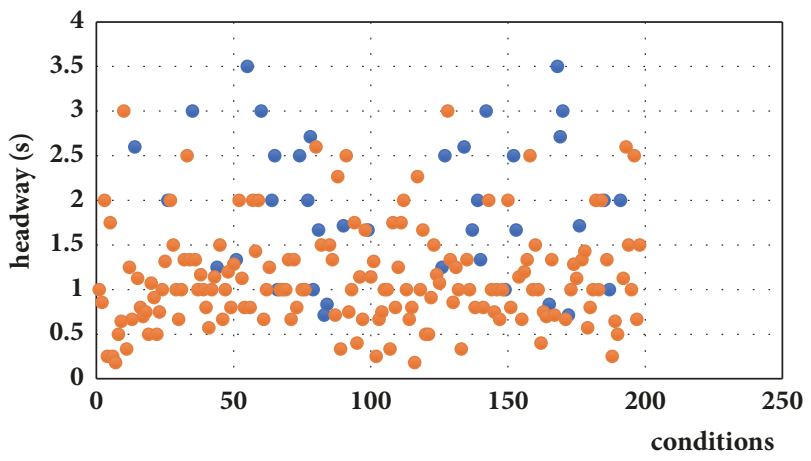

U-turn

- wait

FIGURE 4: Observed game results in different scenes.

not rule-based, it is an uncertain problem, and the vehicles' behavior is the outcome of interaction between each other.

2.3.2. UTG Model Parameter Calibration. We collected statistics for probability of U-turn vehicles' action under two kinds of headway intervals. The results of surveys are shown in Table 3.

We calibrated the model parameters of $a$ and $c$ in (1) based on the survey data. Taking the middle value of each headway interval as the representative headway, this represents the delay of U-turn vehicle; the reciprocal of it represents the threat perception of vehicles. Two sets of different headway intervals can jointly be solved parameters $a$ and $c$, and we obtain the results $a=0.16, c=1.05$.

\section{U-Turn Simulation Model Based on Cellular Automata}

3.1. Assumptions and the Scene of Simulation. A two-way four-lane cellular automata traffic flow model with a U-turn facility in middle of road is established (see Figure 5).

3.1.1. Road Condition Assumptions. (1) Lane A is in the same direction of Lane B, and Lanes C and D are opposite. (2) One cell represents $3.75 \mathrm{~m}$. (3) The road length is set as 200 cells, so the road length equals $750 \mathrm{~m}$.

3.1.2. Operating Conditions Assumptions. (1) The simulation time step is set to 1s. (2) It is assumed that all the vehicles have the same length of 2 cells $(7.5 \mathrm{~m})$ and the maximum speed of 5 cells $\left(v_{\max }=67.5 \mathrm{~km} / \mathrm{h}\right)$. (3) According to the trajectory of the U-turn vehicles in Lanes A and B, the lanes are divided into three areas, $\mathrm{S} 1, \mathrm{~S} 2$, and $\mathrm{S} 3$, wherein $\mathrm{S} 1$ and $\mathrm{S} 3$ are the freerunning sections (S3' length is 100 cells and S1' length is 60 cells); S2 is the U-turn vehicle lane changing area, with the length of 40 cells. The end of S2 is U-turn place (the point is the $100^{\text {th }}$ cell). (4) At the end of S1, a U-turn sign is installed (the point is the $60^{\text {th }}$ cell), and the U-turn vehicle running in the S1 area at Lane A completes the lane change to Lane B in S2 after seeing the U-turn sign. It finally turns around at B1. (5) When the U-turn vehicle in Lane A reaches the U-turn area $\mathrm{A} 0$ or $\mathrm{A} 1$, it will wait at $(\mathrm{A} 0, \mathrm{A1})$ until $(\mathrm{B} 0, \mathrm{~B} 1)$ are available.

U-turn vehicles specific behavior process is as follows: The vehicle completes the U-turn through $(\mathrm{B} 0, \mathrm{~B} 1)$ to $(\mathrm{B} 1, \mathrm{C1})$, $(\mathrm{B} 1, \mathrm{C} 1)$ to $(\mathrm{C} 1, \mathrm{D} 1)$, and $(\mathrm{C} 1, \mathrm{D} 1)$ to $(\mathrm{D} 1, \mathrm{D} 0)$.

3.2. Rule of Vehicle Movement. According to the division of the road area, there are two types of lane changing rules: The forced lane changing rule of the U-turn vehicle in S2 of Lane $A$ and the general lane changing rule of the other vehicles on the road. Using the classic lane changing rule proposed by Chowdhury [37] (which are popularly used in the simulation of traffic flow), we construct the general lane changing rule and the forced lane changing rule.

We use the classic NaSch model as the vehicle update rule. In the NaSch model, time, space, and speed are discretized, which is the minimum model that can reproduce the basic characteristics of road traffic flow. Although the NaSch model [38] has a simple form, it can describe some actual traffic phenomena, such as spontaneous congestion, and time-andstop phenomena under crowded conditions. This is in line with the simulation environment we built, in which the straight vehicles are affected by the vehicle's U-turn and cause traffic congestion. However, it is important to understand that one of the biggest shortcomings of the NaSch model is that it does not reproduce Kerner's three-theory framework $[39,40]$. When using the NaSch model for simulation, traffic flow phenomena such as metastable state and hysteresis cannot be reproduced. Therefore, the simulation results may be affected by the limitations of the NaSch model.

It is also stipulated that the U-turn vehicle on Lane B will no longer change the lane in the area S2 but eventually moves to the U-turn position B1. U-turn vehicle in Lane A, without meeting lane change condition, will wait at Position Al until it can safely change to B and eventually to B1. Combining the above two lane changing rules constitutes the lane changing rule of the model.

The opening boundary conditions are used to control the access of the vehicle, according to the monitor of locations of 


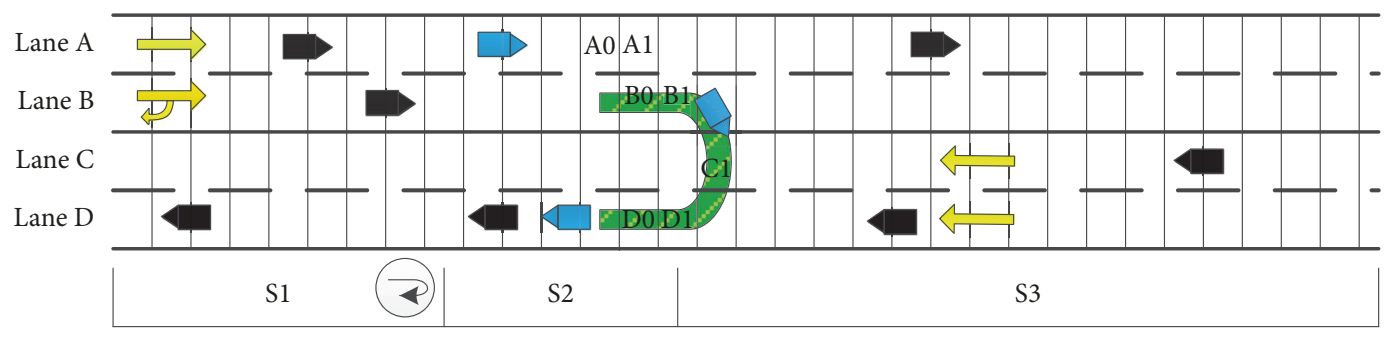

FIGURE 5: Scene of vehicle U-turn cellular automata model.

the head vehicle and the last vehicle. When the head vehicle position is greater than the lane length, it is already out of the road.

Otherwise, when the last vehicle position from the road entrance is greater than $v_{\max }$ cells, it will produce new vehicles into the road with the probability $q$, wherein $x_{\text {last }}$ is the last vehicle's position; $\min \left(x_{\text {last }}-v_{\max }, v_{\max }\right)$ is the new vehicles' position; $v_{\text {max }}$ is its speed.

At the same time, the rule of vehicle into the road is modified: The vehicles into Lanes A and B with probability $q$ will change the type of vehicle into U-turn vehicle with probability $p_{u t u r n}$, into straight vehicle with probability $1-$ $p_{\text {uturn }}$.

3.3. Rule of U-Turn. As stated in Section 2, the U-turn drivers' decisions are deduced from game model. The delays of Uturn vehicle and straight vehicle are described in cellular model.

$t_{1 c}$ and $t_{1 D}$ denote the delays of the U-turn vehicle in Lanes $\mathrm{C}$ and $\mathrm{D}$, respectively:

$$
\begin{gathered}
t_{1 c}=\frac{L_{c}}{v_{\text {chead }}}, \\
t_{1 D}=\frac{L_{D}}{v_{\text {Dhead }}}
\end{gathered}
$$

where $L_{c}=x_{\text {chead }}-x_{c 1}+l_{\text {veh }} ; L_{D}=x_{\text {Dhead }}-x_{D 1}+l_{\text {veh }}$; $v_{\text {chead }} v_{\text {Chead }}, v_{\text {Dhead }}, x_{\text {chead }}$, and $x_{\text {Dhead }}$ are the speeds and the positions of the straight vehicles, closest to the U-turn points $\mathrm{C} 1$ and $\mathrm{D} 1 ; x_{c 1}$ and $x_{D 1}$ are the positions of $\mathrm{C} 1$ and $\mathrm{D} 1 ; l_{v e h}$ is the vehicle length.

$t_{2 c}$ and $t_{2 D}$ denote the delays of the straight vehicles in Lanes C and D, respectively; thus

$$
\begin{aligned}
& t_{2 c}=2+v_{\text {chead }}-\frac{2 L_{1}+\left(1+v_{\text {chead }}\right) v_{\text {chead }}}{2 v_{\text {chead }}} \\
& t_{2 D}=2+v_{\text {Dhead }}-\frac{2 L_{2}+\left(1+v_{\text {Dhead }}\right) v_{\text {Dhead }}}{2 v_{\text {Dhead }}}
\end{aligned}
$$

where $L_{1}=x_{\text {chead }}-x_{c 1}-1$ is the distance between the position of straight vehicle and the $\mathrm{C} 1$ cell; $L_{2}=x_{\text {Dhead }}-x_{D 1}-1$ is the distance between the position of straight vehicle and the D1 cell.

In order to quantify the relationship between the utilities of delay and threat perception, the results are normalized. According to the analysis of the UTG model, the U-turn rule is divided into two states:
(1) When the U-turn gap is greater than 2, that is, $t=$ $L_{\text {head }} / v_{\text {head }}$, the U-turn vehicle does not affect the U-turn area. At this time, the U-turn vehicle passes. $L_{\text {head }}$ indicates the distance from the location of the straight vehicle to the conflicting location; $v_{\text {head }}$ is the speed at this time.

(2) There is a game relationship between the U-turn vehicle and the straight vehicle when the U-turn gap is less than 2; the decision of the drivers is an uncertain problem under this situation. The probability distribution of these two strategies is deduced from gam model.

U-turn vehicle (pass, wait) probability in Lane C is as follows:

$$
\begin{aligned}
& \left(p_{u u}^{c}, p_{u w}^{c}\right) \\
& \quad=\left(\frac{2 a t_{1 c}}{2 k+(1+c) a t_{1 c}}, 1-\frac{2 a t_{1 c}}{2 k+(1+c) a t_{1 c}}\right)
\end{aligned}
$$

Straight vehicle (pass, wait) probability in Lane C is as follows:

$$
\left(p_{s t}^{c}, p_{s w}^{c}\right)=\left(\frac{2 a t_{2 c}}{2 k+(1+c) a t_{2 c}}, 1-\frac{2 a t_{2 c}}{2 k+(1+c) a t_{2 c}}\right)
$$

U-turn vehicle (pass, wait) probability in Lane D is as follows:

$$
\begin{aligned}
& \left(p_{u u}^{D}, p_{u w}^{D}\right) \\
& \quad=\left(\frac{2 t_{1 D}}{2 k+(1+c) a t_{1 D}}, 1-\frac{2 t_{1 D}}{2 k+(1+c) a t_{1 D}}\right)
\end{aligned}
$$

Straight vehicle (pass, wait) probability in Lane D is as follows:

$$
\begin{aligned}
& \left(p_{s t}^{D}, p_{s w}^{D}\right) \\
& \quad=\left(\frac{2 t_{2 D}}{2 k+(1+c) a t_{2 D}}, 1-\frac{2 t_{2 D}}{2 k+(1+c) a t_{2 D}}\right)
\end{aligned}
$$

Here, $a=0.16, c=1.05$ (parameter calibration in Section 2.3). It will have a collision in the condition that both the straight vehicle and the U-turn vehicle choose to pass. In the real life, in order to avoid the collision at this time, the drivers will honk, gesture, and so on, which indicates a second game. In this model, we set the U-turn vehicles pass with probability $p 2=0.5$. 


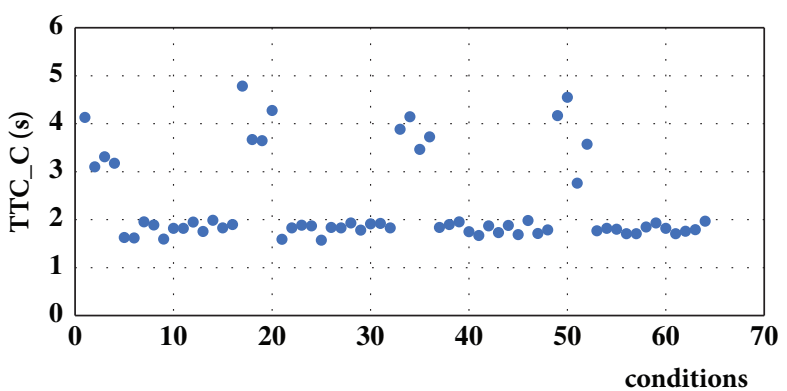

(a)

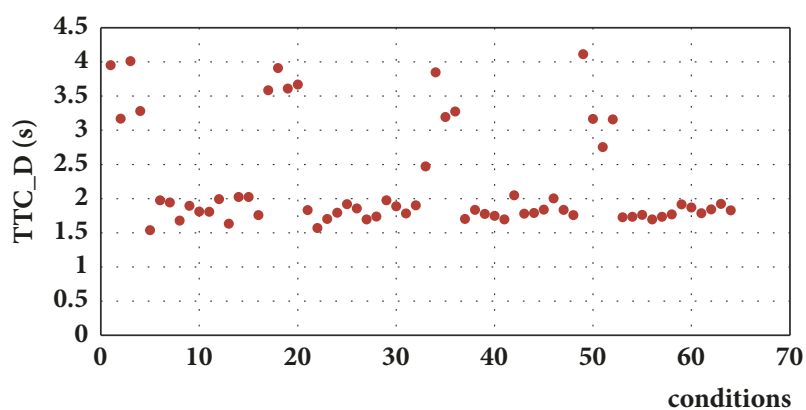

(b)

Figure 6: TTC in lane. (a) Lane C. (b) Lane D.

\section{Simulation Analysis and Discussion}

Parameter setting: (1) Lane changing probability is $p_{\text {change }}=$ 0.55 (using the research by Liu [36]). (2) Random moderation probability is $p_{\text {slow }}=0.2$ based on experience.

By changing the probability of vehicle entering Lanes A and $\mathrm{B}$ (marking the probability as $p_{i n 1}$ ), the probability of vehicle entering Lanes $\mathrm{C}$ and $\mathrm{D}$ (marking the probability as $\left.p_{\text {in } 2}\right)$, and the proportion of the U-turn vehicles in Lanes A and $\mathrm{B}$ (marking the probability as $p_{\text {uturn }}$ ), the impact of the U-turn on the traffic flow is simulated. The set of values of $p_{i n 1}$ and $p_{i n 2}$ is $\{0.2,0.4,0.6,0.8\}$; the set of values of $p_{u t u r n}$ is $\{0,0.2,0.4,0.6,0.8\}$; a total of 80 kinds of road conditions are simulated, simulating 500 time steps in each case.

\subsection{Analysis of Traffic Conflict in U-Turn Area}

4.1.1. Definition of Traffic Conflict and the Division of Severity. The most frequently used conflict indexes are the time of collision (TTC) and the postinvasion time (PET). In the work, the TTC index is selected to evaluate the severity of the Uturn conflict. TTC means that if the current speed, direction, and trajectory are maintained, two road users expect to have a collision. Smaller TTC leads to the more serious conflict.

In order to quantitatively study the severity of the conflict of the U-turn, the conflict was divided into discrete severity levels according to the different thresholds of the conflict index. At the same time, as the conflicts will happen in Lanes $\mathrm{C}$ and $\mathrm{D}$, the severity of the conflict is calculated based on the lane.

Figure 6 shows results, the abscissa includes 64 conditions (except for 16 conditions of no U-turn vehicle), and the ordinate is the value of TTC. It can be seen that the law of changes of each lane TTC in different conditions is similar. Almost all of the TTC values are between 1.5 and 5, and the severity of the conflict according to the TTC value is divided into three levels-severe [1.5-2], slight [2-3], and potential [3-5]. Corresponding to this, a cellular automaton by the discriminant method is as follows.

Severe: The average time for the straight vehicle to arrive at the conflict point is between 1.5 and 2 time steps. The Uturn vehicle takes up two cells, at least 2 time steps to leave the area without stopping. It means that the U-turn vehicle and the straight vehicle will inevitably change their behavior strategy because of conflict; if not, it will inevitably produce traffic accidents. The severity of the conflict is defined as "severe" in this condition.

Slight: The average time for the straight vehicle to arrive at the conflict point is between 2 and 3 time steps. In this condition, if the U-turn vehicle does not stop to Uturn directly, the straight vehicle do not have to change the behavior. If the U-turn vehicle needs to wait for a peripheral cause (for example, the U-turn vehicle in Lane $\mathrm{C}$ is waiting for the pass of the straight vehicle in Lane D), the straight vehicle needs to change its behavior to avoid the collision. The severity of the conflict is defined as "slight" in this condition.

Potential: The average time for the straight vehicle to arrive at the conflict point is more than 3 time steps, meaning that this time can meet the certain demand of the U-turn, and the straight vehicles do not need to change their behavior. However, there will be a conflict if the U-turn vehicle keeps waiting in the conflict point. The severity of the conflict is defined as "potential" in this condition.

4.1.2. Analysis of Influencing Factors. Using SPSS to analyze the influencing factors of traffic conflicts, Table 4 shows the results. According to the significance and correlation coefficient, it can be seen that the impact of $p_{\text {in } 2}$ on the TTC value of Lanes $C$ and $D$ is most significant. The reason is that the size of $p_{\text {in } 2}$ determines the size of the gap when the U-turn vehicle arrives at U-turn area.

We analyze the impact of $p_{i n 2}$ and $p_{\text {uturn }}$ on TTC value of Lane C, as well as the impact of $p_{i n 2}$ and $p_{i n 1}$ on the TTC value of Lane D (see Figure 7).

Analysis of Influencing Factors for TTC in Lane C. Figure 7(a) shows that TTC is influenced by $p_{u t u r n}$ when $p_{\text {in } 2}$ is a small value (such as $p_{i n 2}=0.2$ ). A larger $p_{\text {uturn }}$ results in the smaller TTC value. The reason is that the straight traffic flow shows a free-flow condition when $p_{i n 2}$ is small. In this condition, more $\mathrm{U}$-turn vehicles lead to bigger possibility of U-turn vehicle in a small gap as well as the smaller TTC value.

The TTC value and $p_{i n 2}$ show the relationship of the open U-shaped curve when $p_{\text {uturn }}$ is fixed, and the lowest TTC value appears in the vicinity of $p_{i n 2}=0.5$. The size of the TTC value has a strong sensitivity with the change of $p_{i n 2}$ value when $p_{\text {in } 2}<0.5$; the TTC value is near 4 when $p_{\text {in } 2}=0.2$; the 


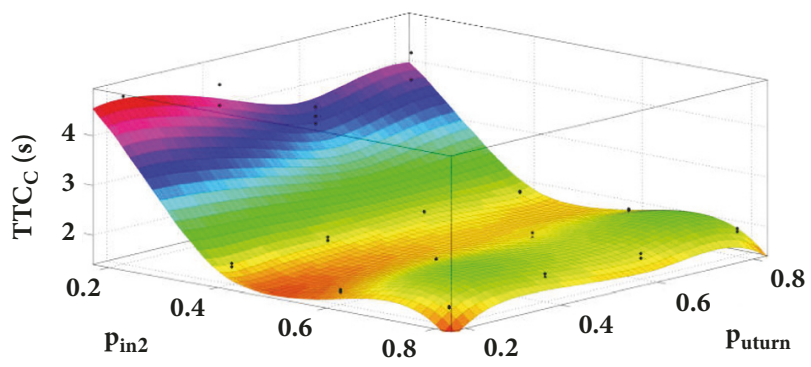

(a)

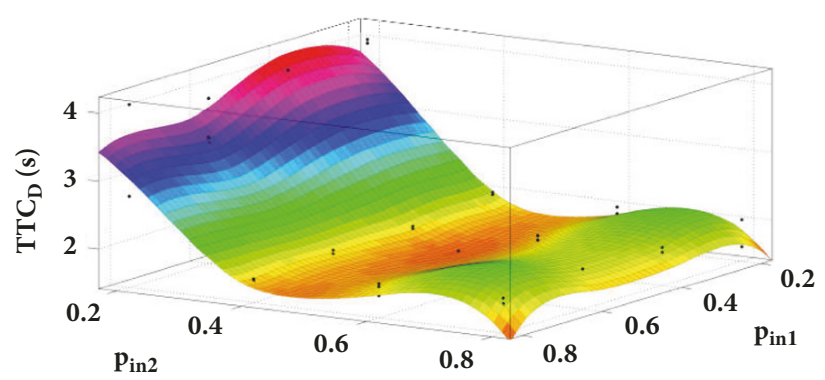

(b)

FIgURE 7: Impact of factors on TTC in lane. (a) Lane C. (b) Lane D.

TABLE 4: Correlation matrix for conflict.

\begin{tabular}{|c|c|c|c|c|}
\hline & & $p_{i n 1}$ & $p_{i n 2}$ & $p_{\text {uturn }}$ \\
\hline \multirow{2}{*}{ TC of lane $\mathrm{C}$} & Correlation coefficient & -0.045 & -0.548 & 0.241 \\
\hline & Significance & 0.722 & 0.000 & 0.055 \\
\hline \multirow{2}{*}{ TTC of lane D } & Correlation coefficient & -0.097 & -0.415 & -0.012 \\
\hline & Significance & 0.447 & 0.001 & 0.924 \\
\hline
\end{tabular}

TTC value drops rapidly to 2 when $p_{\text {in } 2}$ increases from 0.2 to 0.4 ; the TTC value changes little in a severe condition when $p_{\text {in } 2}>0.5$.

The reason for the above phenomenon is related to the traffic flow of Lane $\mathrm{C}$ affected by $p_{\text {in } 2}$. The traffic flow of Lane $\mathrm{C}$ is in the free-flow condition when $p_{i n 2}$ is small. The Uturn gap decreases as $p_{i n 2}$ increases; that is, the TTC value decreases as $p_{\text {in } 2}$ increases. With the increase of $p_{i n 2}$, the road shows a stable crowded flow. U-turn gap size tends to be stable, and the range of TTC value shows stability. Correspondingly, TTC has been in a severe condition when $p_{\text {in } 2}>0.5$.

Analysis of Influencing Factors for TTC in Lane D. Figure 7(b) shows that the trend of TTC in Lane D is the same as that of Lane $\mathrm{C}$, except that the minimum TTC value of $\mathrm{D}$ is near $p_{\text {in2 }}(0.4)$, and the minimum TTC value of Lane $C$ is near $p_{\text {in } 2}(0.5)$. The reason is that the U-turn vehicle needs to be accelerated in $\mathrm{D}$ after completion of the U-turn, resulting in a greater delay for the straight vehicle in $\mathrm{D}$. It can cause a greater traffic density in the U-turn area. The U-turn gap is smaller, so that Lane $C$ appears with the minimum TTC value when $p_{\text {in } 2}=0.5$. However, $\mathrm{D}$ appears with the minimum TTC value when $p_{i n 2}=0.4$.

\subsection{Analysis of Delay on the Road}

4.2.1. Characterization Method of Delay. The U-turn of the vehicle at the U-type facility will result in the delay of the same/opposite way vehicles. The former is caused by the lane changing behavior in interweaving zone of S2 area, and the latter is caused by the conflict with U-turn vehicle.

In the work, based on the theory of traffic wave, the influence of U-turn on delay is analyzed by comparing the change of traffic flow in U-turn area. Since the steering area has a significant delay on the traffic flow of the road, only the upper steering area of each lane is considered in the statistics (see Figure 8). The $\mathrm{X}$ axis represents 80 kinds of situations, and the $\mathrm{Y}$ axis represents the traffic volume. It can be seen that the traffic volumes of Lanes A, B/C, and D are similar in different situations. The reason is that the vehicle is allowed to change lane between the same lanes, which makes the traffic flow in the same lanes relatively average.

4.2.2. Analysis of Influencing Factors. Using SPSS to analyze the factors influencing the traffic volume, Table 5 shows the results. The impact of $p_{\text {uturn }}$ on the traffic volume for 4 lanes is significant, so is the impact of $p_{i n 2}$ on the traffic volume for Lanes $\mathrm{C}$ and $\mathrm{D}$.

We analyze the impact of $p_{\text {uturn }}$ and $p_{\text {in } 1}$ on traffic volume for Lanes A and B. The traffic volume situation of A is similar to that of $\mathrm{B}$, so the traffic volume of $\mathrm{A}$ is selected as an example. We also analyze the impact of $p_{\text {uturn }}$ and $p_{\text {in } 2}$ on traffic volume for the Lanes $C$ and $D$. The traffic volume situation of $\mathrm{C}$ is similar to that of $\mathrm{D}$, so the traffic volume of $\mathrm{C}$ is selected as an example. Figure 9 shows the results.

(1) Analysis of Influencing Factors for Delay in Lane A. Figure 9 (a) shows, according to the size of $p_{\text {in } 1}$, the impact of $p_{\text {uturn }}$ on the traffic volume, which is divided into two aspects.

By comparing the traffic volume with $p_{\text {uturn }}=0$, when $p_{\text {in } 1}<0.3$, there is little impact of $p_{u t u r n}$ on traffic volume. The reason is that in the case of a small size of $p_{i n 1}$, the Uturn gap is big when the U-turn vehicle reaches the U-turn area. The U-turn does not affect the normal running of the straight vehicle, so the traffic volume does not change much.

When $p_{\text {in } 1}>0.3$, the size of the traffic volume is significantly affected by $p_{u t u r n}$, and the size of the traffic volume decreases with the increase of $p_{\text {uturn }}$. On both sides of $p_{\text {uturn }}(0.2)$, the rate of traffic volume change is drastically 


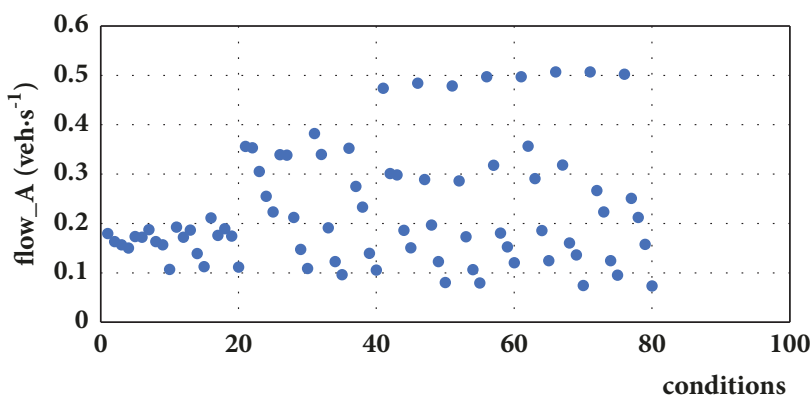

(a)

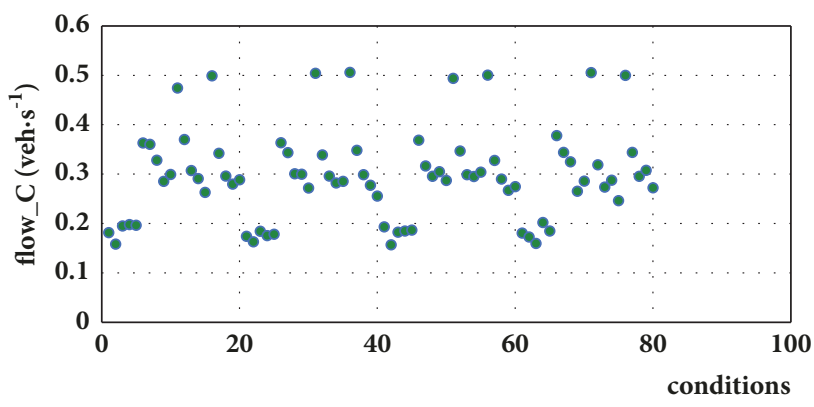

(c)

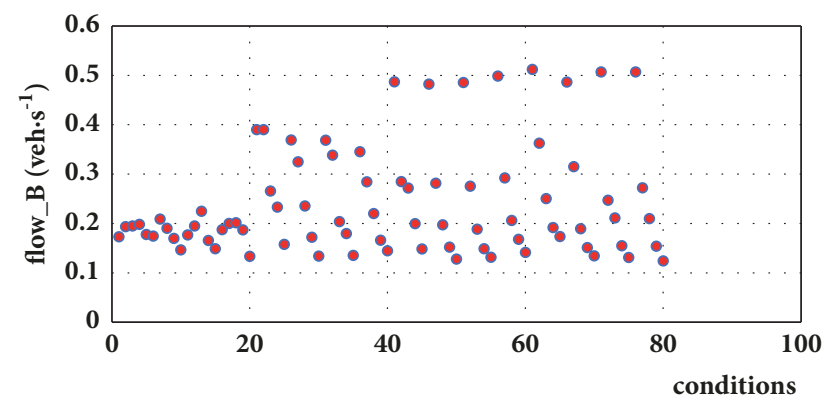

(b)

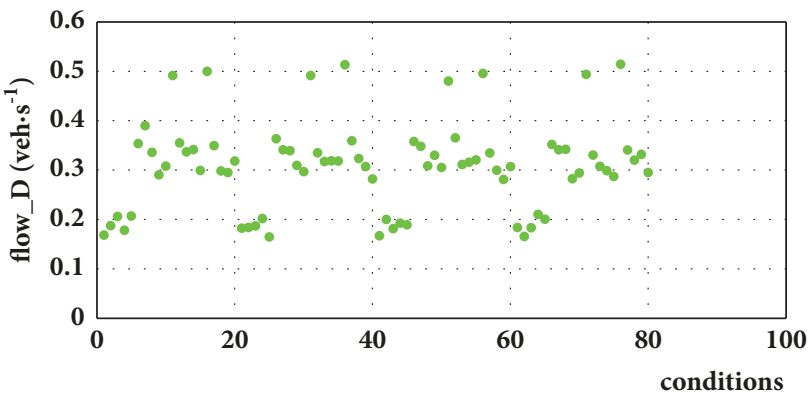

(d)

Figure 8: Flow in lane. (a) Lane A, (b) Lane B, (c) Lane C, and (d) Lane D.

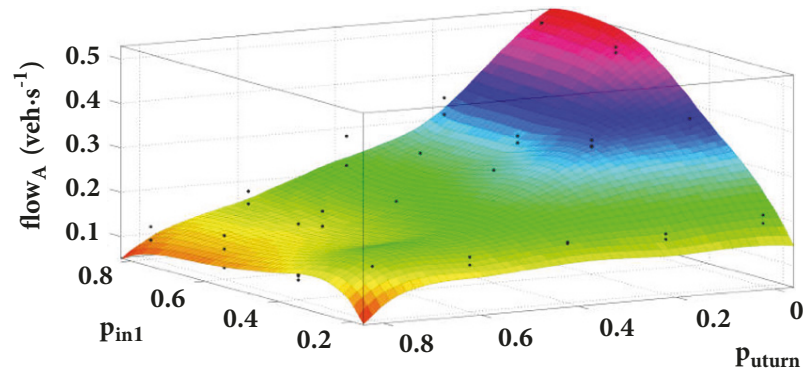

(a)

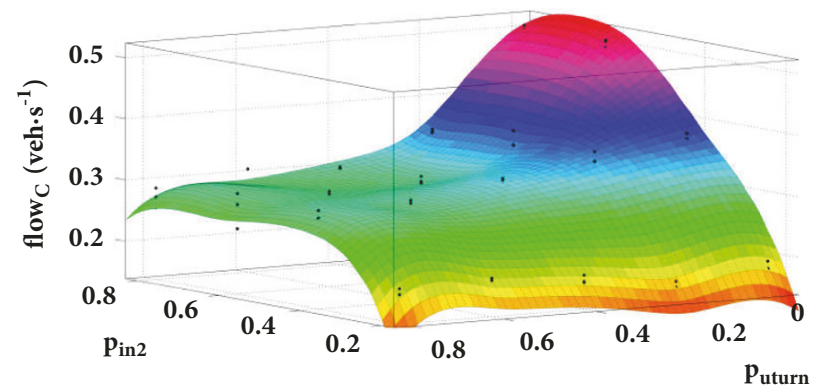

(b)

Figure 9: Impact of factors on volume in lane. (a) Lane A. (b) Lane C.

TABLE 5: Correlation matrix of influencing factors for delay.

\begin{tabular}{lcccc}
\hline & & $p_{\text {in } 1}$ & $p_{\text {in } 2}$ & $p_{\text {uturn }}$ \\
\hline \multirow{2}{*}{ Lane A } & Correlation coefficient & 0.171 & -0.127 & -0.828 \\
& Significance & 0.130 & 0.262 & $\mathbf{0 . 0 0 0}$ \\
\hline \multirow{2}{*}{ Lane B } & Correlation coefficient & 0.160 & -0.122 & -0.829 \\
& Significance & 0.157 & 0.281 & 0.554 \\
\multirow{2}{*}{ Lane C } & Correlation coefficient & -0.039 & $\mathbf{0 . 0 0 0}$ \\
& Significance & 0.731 & 0.000 & $\mathbf{0 . 0 0 0}$ \\
\hline \multirow{2}{*}{ Lane D } & Correlation coefficient & -0.059 & $\mathbf{0 . 0 0 0}$ & -0.448 \\
& Significance & 0.606 & $\mathbf{0 . 0 0 0}$ \\
\hline
\end{tabular}

different, and the rate of change is less when $p_{\text {uturn }}>0.2$ than that when $p_{\text {uturn }}<0.2$. It is verified that the existence of the U-turn area will lead to a traffic phase translation obtained by Combinido [21] et al. It occurs after $p_{i n 1}>0.3$, that is, the large road traffic volume occurs in the scenario.
(2) Analysis of Influencing Factors for Delay in Lane C. As shown in Figure 9(b), according to the size of $p_{\text {in } 2}$, the impact of puturn on road traffic volume is divided into two aspects. The law of impact is similar to that of Lane A, but the following points are different. 


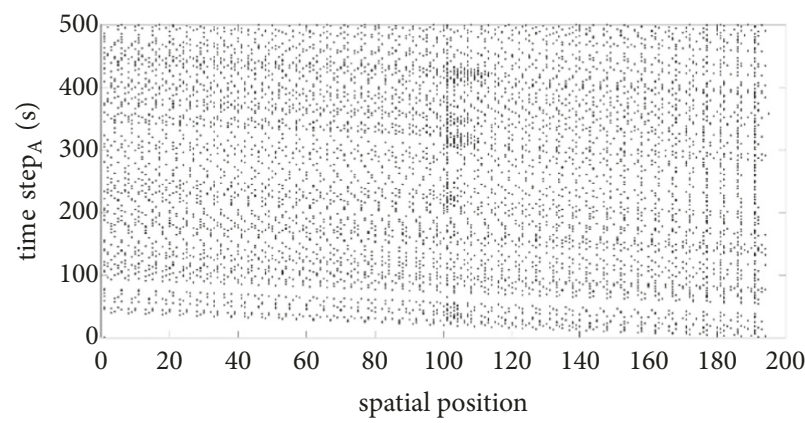

(a)

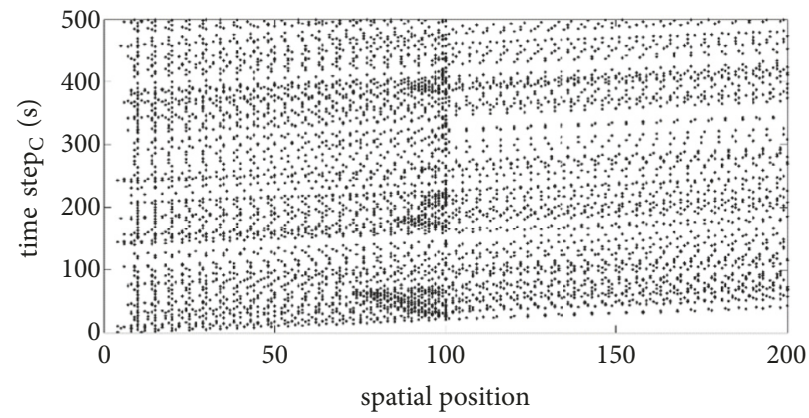

(c)

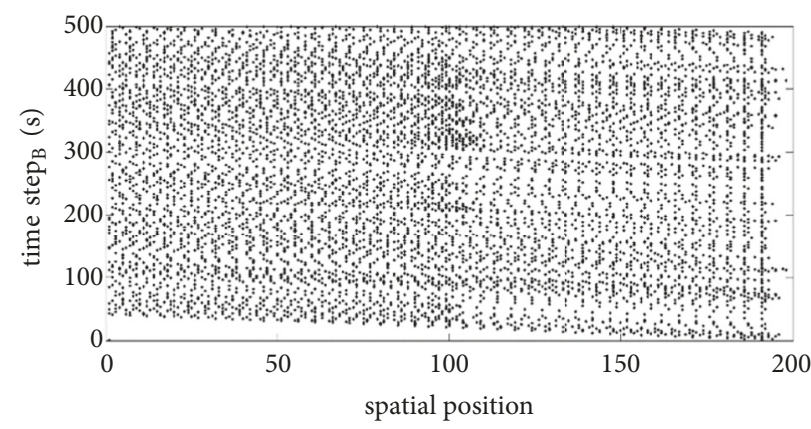

(b)

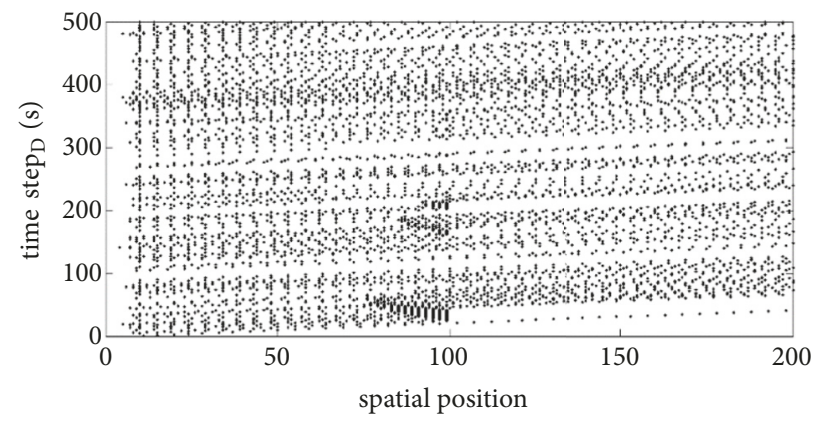

(d)

FIGURE 10: Space-time diagram in lane when $p_{i n 1}=0.3 p_{i n 2}=0.3$; and $p_{\text {uturn }}=0.2$. (a) Lane A, (b) Lane B, (c) Lane C, and (d) Lane D.

In the case of $p_{i n 2}>0.3$, the traffic volume of $\mathrm{C}$ is different from that of A. Meanwhile, the traffic volume of $\mathrm{C}$ is down firstly and then remains the same. That is, when $p_{i n 2}>0.3$, the presence of the U-turn will change traffic flow from free to crowded.

The traffic volume of $\mathrm{C}$ forms a crowded flow in the vicinity of $p_{u t u r n}=0.3$, and that of $\mathrm{A}$ forms a crowded flow in the vicinity of $p_{u t u r n}=0.1$. It indicates that the impact of $p_{u t u r n}$ on the same way traffic volume is significantly greater than that on the straight traffic volume. The reason is that there is no U-turn waiting area between the lanes in the established model; that is, the U-turn vehicle can only wait in Lane B, which will affect the passage of the same way of straight vehicle. However, when the U-turn vehicle is over a certain number and the U-turn waiting area cannot meet the waiting position of all $U$-turn vehicles, the $U$-turn should still wait in B regardless of whether there exists a U-turn waiting area or not.

When the U-turn vehicle in A and B is queued, it will obviously block the straight travel of these lanes, and this delay will spread in the form of waves upstream, resulting in continuous decrease of traffic flow. For $\mathrm{C}$ and $\mathrm{D}$, the Uturn vehicles can U-turn in U-turn gap; although affecting the driving of the straight vehicles, they will not cause the stairs of the flow, so traffic volume will not be reduced to a minimum, but in the state of crowded flow.

4.3. Discussion. We can get the following conclusions by analyzing conflict and delay.
(1) In the case of conflict, the TTC value is highly sensitive to $p i n 2$, and the TTC value is greater than 3 when $p_{i n 2}<0.3$. The severity of the conflict becomes potential.

(2) In the case of delay, the presence of U-turn vehicles will significantly affect the size of road traffic volume when the probability of entering vehicle is large, and the critical size of its traffic phase transition is near puturn (0.2).

Thus, $p_{\text {in } 1}=0.3 ; p_{\text {in } 2}=0.3 ; p_{\text {uturn }}=0.2$. It is a critical state of the U-turn conflict and the traffic delay of the road section. The presence of the $\mathrm{U}$-turn vehicle in this state does not have a significant effect on the traffic flow.

Figure 10 shows the time and space distribution of the traffic flow in this critical state, and $\mathrm{X}$ axis is the spatial position. The vehicles in Lanes A and B are from left to right, and those in Lanes $\mathrm{C}$ and $\mathrm{D}$ are in the contrary driving direction. The central location (that is 100) is the U-turn area. At this time, the road traffic flow is just affected by the Uturn vehicles; in the upper reach of each lane, a certain range of crowded traffic is formed, without affecting the statues of the entire traffic flow.

\section{Conclusions}

In order to analyze the traffic conflict caused by vehicle $\mathrm{U}$ turning and its influence on traffic flow of road section, we establish the UTG model to describe drivers' interactions between U-turn vehicle and straight vehicle and run simulation under different traffic condition based on 
cellular automata. Our works are mainly summarized in three aspects:

(1) Considering the uncertainty of the drivers' decisionmaking, the work uses the game theory to analyze the interaction between $U$-turn vehicle driver and straight vehicle driver and constructs the UTG model. Here, traffic delay and threat perception are considered as the factors of the game. Finally, the mixed strategy is obtained; the parameters are verified.

(2) The U-turn simulation model is constructed based on cellular automata. On the basis of two-way four-lane road, the rule of lane changing was modified into two forms: The general lane changing rule and the forced lane changing rule. The rule of updating was modified to generate two types of vehicles: the U-turn vehicle and the straight vehicle. Besides, the rules of U-turning behaviors are defined according to the UTG model.

(3) The influencing factors are identified with their correlation analyzed by numerical simulation of different traffic conditions. The impact of the U-turn vehicle on the traffic flow is analyzed from two aspects: traffic delay and traffic conflict. From the perspective of traffic delay, the arriving rate of the U-turn vehicles affected the traffic flow of the whole road section, and its influence degree was Lanes A and $\mathrm{B}>$ Lanes $\mathrm{C}$ and $\mathrm{D}$. From the perspective of the conflict, the severity of conflict is classified and the cause is identified by analyzing the arrival rate of the U-turn vehicle and the conflicting straight vehicle and the relationship with one another. The opening of the U-turn area should focus on the size of the straight traffic flow.

This paper makes a useful attempt to construct the U-turn model by considering the driver's decision-making uncertainty and analyze the effect of U-turn behavior on traffic through the construction of cellular automata simulation. This work provides some information for traffic managers to choose the opening for U-turn vehicles in road section. Due to the difficulty in exploring drivers psychological aspect from video data, the parameters in model can be verified by other approaches (e.g., questionnaires or driving simulator). Further research can also be made on the identification of the impact of U-turn traffic on the different lanes including the same direction lanes which will be influenced by the lane change behaviors of U-turn vehicles. Moreover, some parameters such as the size of the opening and the form of the waiting area which are important to the U-turn behavior of vehicles will been considered.

\section{Data Availability}

The data used to support the findings of this study are included within the article.

\section{Conflicts of Interest}

The authors declared no potential conflicts of interest with respect to the research, authorship, and/or publication of this article.

\section{Acknowledgments}

This work was supported by the National Key Research and Development Program of China [Grant no. 2016YFB0101601] and Jilin Transportation and Transportation Science and Technology Project [2018-1-19].

\section{References}

[1] B. Liu, Q. Sun, and A. El Kamel, "Improving the Intersection's Throughput using V2X Communication and Cooperative Adaptive Cruise Control," IFAC-PapersOnLine, vol. 49, no. 5, pp. 359-364, 2016.

[2] W. W. Hunter, J. R. Feaganes, and R. Srinivasan, "Conversions of wide curb lanes: The effect on bicycle and motor vehicle interactions," Transportation Research Record Journal of the Transportation Research Board, vol. 1939, no. 1, pp. 37-44, 2005.

[3] A. P. Silvano, H. N. Koutsopoulos, and X. Ma, "Analysis of vehicle-bicycle interactions at unsignalized crossings: A probabilistic approach and application," Accident Analysis \& Prevention, vol. 97, pp. 38-48, 2016.

[4] K. Suzuki and H. Ito, "Empirical Analysis on Risky Behaviors and Pedestrian-Vehicle Conflicts at Large-Size Signalized Intersections," pp. 2144-2157.

[5] R. Almodfer, S. Xiong, Z. Fang, X. Kong, and S. Zheng, "Quantitative analysis of lane-based pedestrian-vehicle conflict at a non-signalized marked crosswalk," Transportation Research Part F: Traffic Psychology and Behaviour, vol. 42, pp. 468-478, 2015.

[6] M. Iryo-Asano and W. Alhajyaseen, "Consideration of a Pedestrian Speed Change Model in the Pedestrian-Vehicle Safety Assessment of Signalized Crosswalks," Transportation Research Procedia, vol. 21, pp. 87-97, 2017.

[7] H. Iasmin, A. Kojima, and H. Kubota, "Safety effectiveness of pavement design treatment at intersections: Left turning vehicles and pedestrians on crosswalks," IATSS Research, vol. 40, no. 1, pp. 47-55, 2016.

[8] Y. Hashimoto, Y. Gu, L.-T. Hsu, M. Iryo-Asano, and S. Kamijo, "A probabilistic model of pedestrian crossing behavior at signalized intersections for connected vehicles," Transportation Research Part C: Emerging Technologies, vol. 71, pp. 164-181, 2016.

[9] B. R. Kadali and P. Vedagiri, "Proactive pedestrian safety evaluation at unprotected mid-block crosswalk locations under mixed traffic conditions," Safety Science, vol. 89, pp. 94-105, 2016.

[10] H.-T. Zhao, S. Yang, and X.-X. Chen, "Cellular automata model for urban road traffic flow considering pedestrian crossing street," Physica A: Statistical Mechanics and its Applications, vol. 462, pp. 1301-1313, 2016.

[11] X. Li and J.-Q. Sun, "Effects of vehicle-pedestrian interaction and speed limit on traffic performance of intersections," Physica A: Statistical Mechanics and its Applications, vol. 460, pp. 335347,2016

[12] Q. Chen and Y. Wang, "Cellular automata (CA) simulation of the interaction of vehicle flows and pedestrian crossings on urban low-grade uncontrolled roads," Physica A: Statistical Mechanics and its Applications, vol. 432, pp. 43-57, 2015.

[13] J. Hariyono and K.-H. Jo, "Detection of pedestrian crossing road: A study on pedestrian pose recognition," Neurocomputing, vol. 234, pp. 144-153, 2017. 
[14] E. J. Lee, B. C. Ko, and J.-Y. Nam, "Recognizing pedestrian's unsafe behaviors in far-infrared imagery at night," Infrared Physics \& Technology, vol. 76, pp. 261-270, 2016.

[15] J.-E. Källhammer, K. Smith, and P. Matsangas, "Modeling ratings of in-vehicle alerts to pedestrian by leveraging field operational tests data in a controlled laboratory study," Transportation Research Part F: Traffic Psychology and Behaviour, vol. 46, pp. 413-425, 2017.

[16] I. P. Meel, U. Brannolte, D. Satirasetthavee, and K. Kanitpong, "Safety impact of application of auxiliary lanes at downstream locations of Thai U-turns," IATSS Research, vol. 41, no. 1, pp. 1-11, 2017.

[17] Rhaptyalyani, "Evaluation of The Performance of U -Turn Movement Using Vissim Simulation Program; A Case Study At Burlian Road,Palembang, Indonesia," in Proceedings of the Procedding of 15th FSTPT symposium Transportation Forum, Bekasi, Indonesia, 2012.

[18] R. H. Della, J. Arliansyah, and R. Artiansyah, “Traffic Performance Analysis of u-turn and Fly Over u-turn Scenario; A Case Study at Soekarno Hatta Road, Palembang, Indonesia," Procedia Engineering, vol. 125, pp. 461-466, 2015.

[19] R. Olarte, J. G. Bared, L. F. Sutherland, and A. Asokan, "Density models and safety analysis for rural unsignalised restricted crossing U-turn intersections," Procedia - Social and Behavioral Sciences, vol. 16, no. 16, pp. 718-728, 2012.

[20] P. Liu, J. J. Lu, and H. Chen, "Safety effects of the separation distances between driveway exits and downstream U-turn locations," Accident Analysis \& Prevention, vol. 40, no. 2, pp. 760-767, 2008.

[21] L. A. Petrosjan and V. V. Mazalov, Game Theory and Applications, Nova Science Publishers, 2003.

[22] T. Bjørnskau, "The Zebra Crossing Game - Using game theory to explain a discrepancy between road user behaviour and traffic rules," Safety Science, vol. 92, pp. 298-301, 2017.

[23] A. Talebpour, H. S. Mahmassani, and S. H. Hamdar, "Modeling lane-changing behavior in a connected environment: A game theory approach," Transportation Research Part C: Emerging Technologies, vol. 59, pp. 216-232, 2015.

[24] S. Lozano, P. Moreno, B. Adenso-Díaz, and E. Algaba, "Cooperative game theory approach to allocating benefits of horizontal cooperation," European Journal of Operational Research, vol. 229, no. 2, pp. 444-452, 2013.

[25] M. Wang, S. P. Hoogendoorn, W. Daamen, B. van Arem, and R. Happee, "Game theoretic approach for predictive lanechanging and car-following control," Transportation Research Part C: Emerging Technologies, vol. 58, pp. 73-92, 2015.

[26] J. S. L. Combinido and M. T. Lim, "Modeling U-turn traffic flow," Physica A: Statistical Mechanics and its Applications, vol. 389, no. 17, pp. 3640-3647, 2010.

[27] H. W. Guo, Z. Y. Gao, and D. F. Xie, "The influence of U-turn movement on the dynamics of traffic flow," Acta Physica Sinica, vol. 60, no. 5, Article ID 058902-2592, 2011.

[28] C. Hyden, The Development of a Method for Traffic Safety Evaluation: The Swedish Traffic Conflicts Technique, Bulletin Lund Institute of Technology Department, 1987.

[29] J. Archer, Indicators for Traffic Safety Assessment and Prediction and Their Application in Micro-Simulation Modelling: A Study of Urban and Suburban Intersections, 2005.

[30] S. Malkhamah, M. Tight, and F. Montgomery, "The development of an automatic method of safety monitoring at Pelican crossings," Accident Analysis \& Prevention, vol. 37, no. 5, pp. 938-946, 2005.
[31] H. Hiyoshi, Y. Tanioka, T. Hamamoto, K. Matsumoto, and K. Chiba, "Pedestrian movement model based on voronoi cellular automata," Transportation Research Procedia, vol. 2, pp. 336343, 2014.

[32] N. P. Belz, L. Aultman-Hall, and J. Montague, "Influence of priority taking and abstaining at single-lane roundabouts using cellular automata," Transportation Research Part C: Emerging Technologies, vol. 69, pp. 134-149, 2016.

[33] X. Ruan, J. Zhou, H. Tu, Z. Jin, and X. Shi, “An improved cellular automaton with axis information for microscopic traffic simulation," Transportation Research Part C: Emerging Technologies, vol. 78, pp. 63-77, 2017.

[34] A. Yamauchi, J. Tanimoto, A. Hagishima, and H. Sagara, "Dilemma game structure observed in traffic flow at a 2-to-1 lane junction," Physical Review E Statistical Nonlinear \& Soft Matter Physics, vol. 79, no. 2, Article ID 036104, 2009.

[35] J. Tanimoto, S. Kukida, and A. Hagishima, "Social dilemma structures hidden behind traffic flow with lane changes," Journal of Statistical Mechanics Theory and Experiment, vol. 2014, no. 7, Article ID P07019, 2014.

[36] Y. Iwamura and J. Tanimoto, "Complex traffic flow that allows as well as hampers lane-changing intrinsically contains socialdilemma structures," Journal of Statistical Mechanics Theory \& Experiment, vol. 2018, no. 2, Article ID 023408, 2018.

[37] D. Chowdhury, D. E. Wolf, and M. Schreckenberg, "Particle hopping models for two-lane traffic with two kinds of vehicles: effects of lane-changing rules," Physica A: Statistical Mechanics and its Applications, vol. 235, no. 3-4, pp. 417-439, 1997.

[38] K. Nagel and M. Schreckenberg, "A cellular automaton model for freeway traffic," Journal de Physique I, vol. 2, no. 12, pp. 22212229, 1992.

[39] B. Kerner, The Physics of Traffic: Empirical Freeway Pattern Features, Engineering Applications and Theory, Springer, New York, NY, USA, 2004.

[40] S. Kokubo, J. Tanimoto, and A. Hagishima, "A new Cellular Automata Model including a decelerating damping effect to reproduce Kerner's three-phase theory," Physica A: Statistical Mechanics and its Applications, vol. 390, no. 4, pp. 561-568, 2011. 


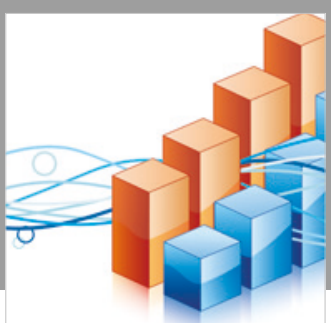

Advances in

Operations Research

\section{-n-m}
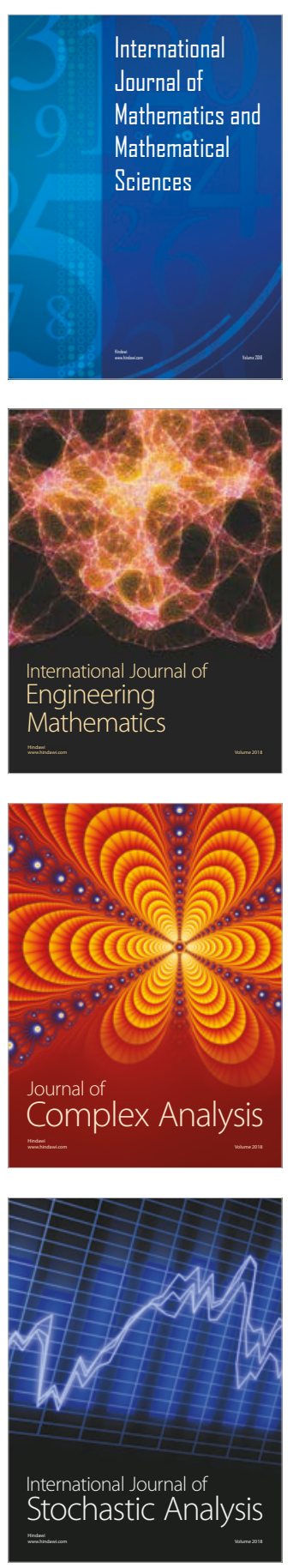
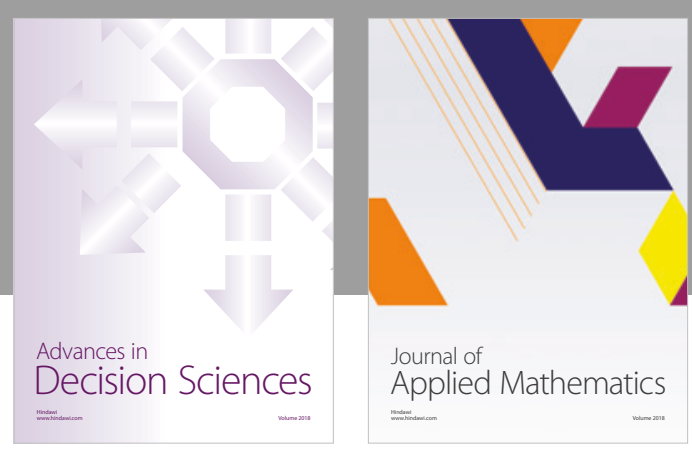

Journal of

Applied Mathematics
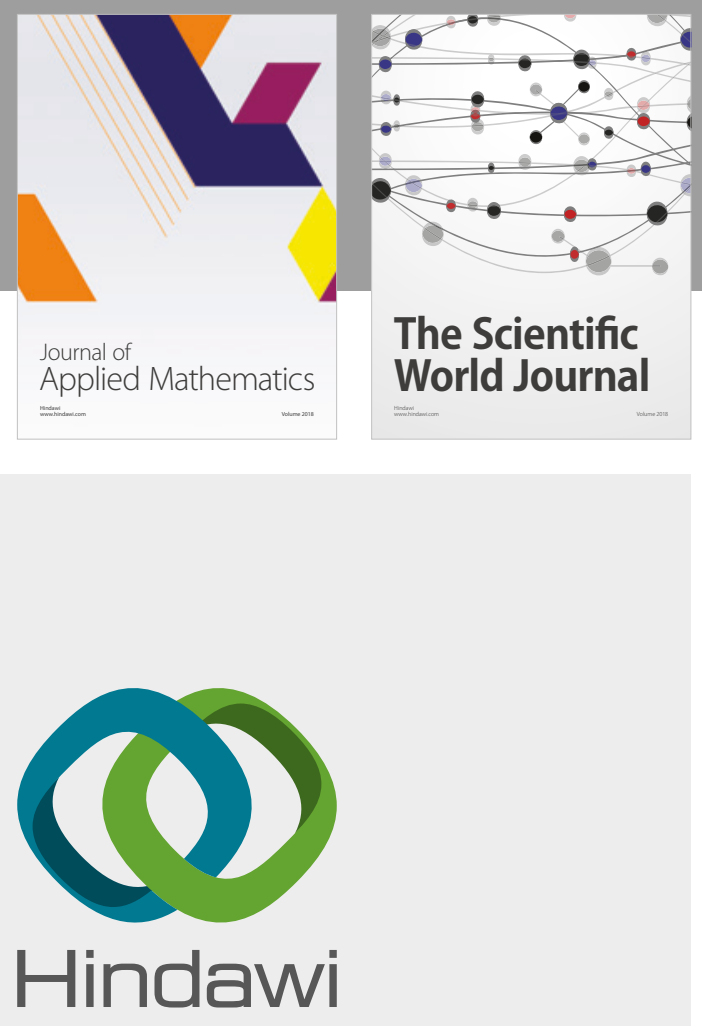

Submit your manuscripts at

www.hindawi.com

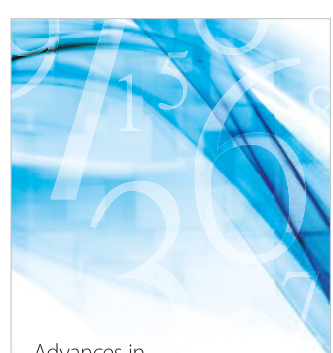

Advances in
Numerical Analysis
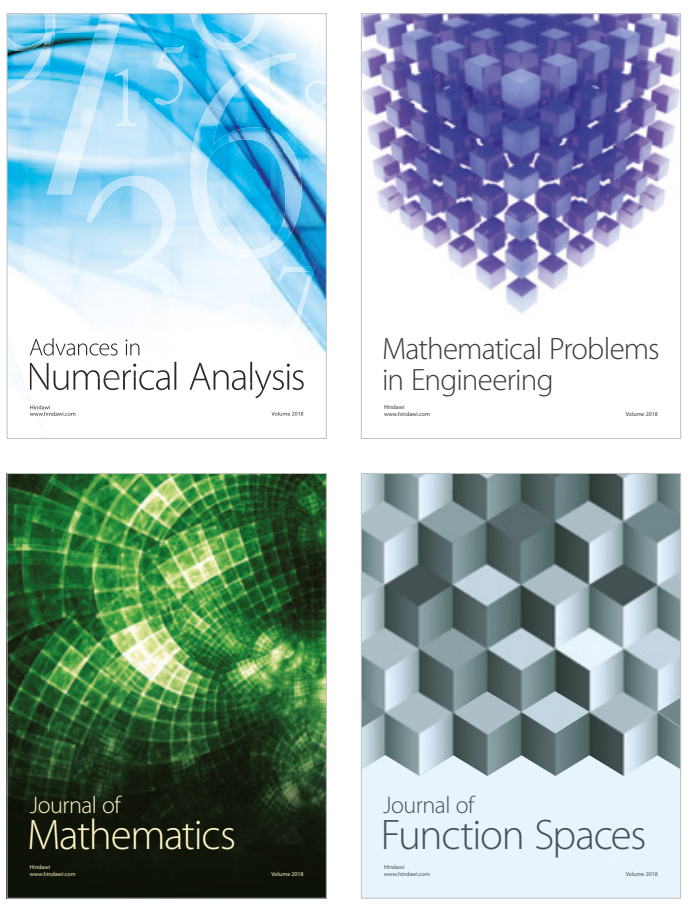

Mathematical Problems in Engineering

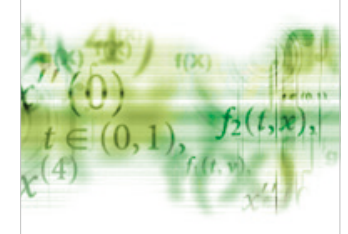

International Journal of

Differential Equations

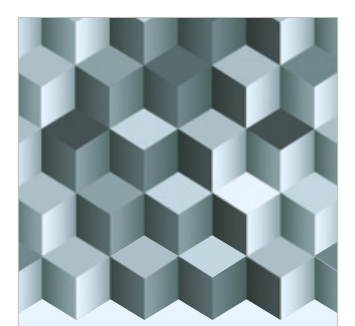

Journal of

Function Spaces

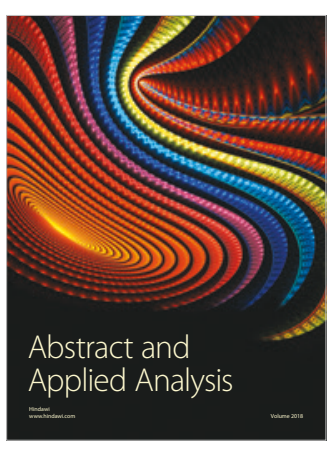

The Scientific

World Journal

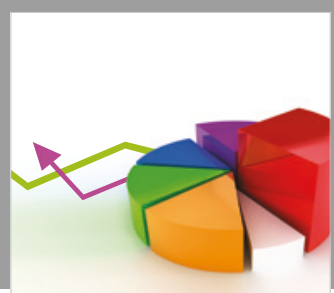

Journal of

Probability and Statistics
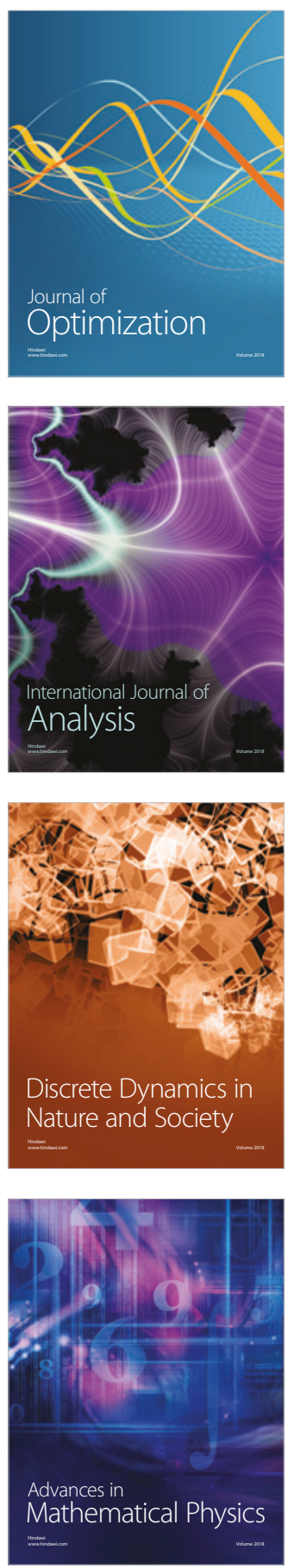